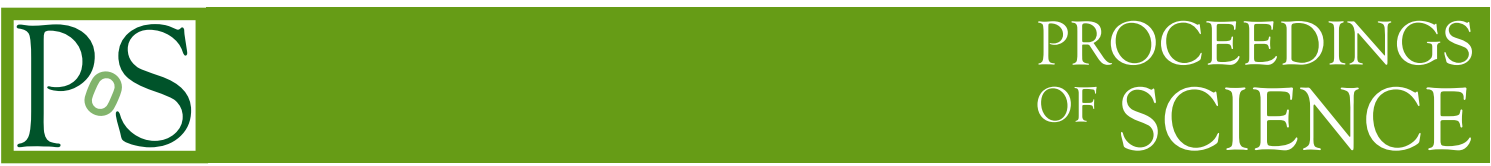

\title{
The Redshift Distribution of the TOUGH Survey
}

\section{P. Jakobsson, ${ }^{a}$ J. Hjorth, ${ }^{b}$ D. Malesani, ${ }^{b}$ R. Chapman, ${ }^{a c}$ J. P. U. Fynbo, ${ }^{b}$ N. R. Tanvir, ${ }^{d}$ B. Milvang-Jensen ${ }^{b}$ and T. Krühler ${ }^{b}$}

${ }^{a}$ Centre for Astrophysics and Cosmology, Science Institute, University of Iceland, Dunhagi 5, 107 Reykjavík, Iceland

${ }^{b}$ Dark Cosmology Centre, Niels Bohr Institute, University of Copenhagen, Juliane Maries Vej 30, 2100 Copenhagen Ø, Denmark

${ }^{c}$ Centre for Astrophysics Research, University of Hertfordshire, Hatfield, Herts AL10 9AB, UK

${ }^{d}$ Department of Physics and Astronomy, University of Leicester,

University Road, Leicester, LE1 7RH, UK

\begin{abstract}
We present the redshift results from a Very Large Telescope (VLT) program aimed at optimizing the legacy value of the Swift mission: to characterize a homogeneous, X-ray selected, sample of 69 GRB host galaxies. 15 new redshifts have been secured, resulting in a 77\% (53/69) redshift completion, making the survey the most comprehensive in terms of redshift completeness of any sample to the full Swift depth, analyzed to date. We present the cumulative redshift distribution and derive a conservative, yet small, associated uncertainty. We constrain the fraction of Swift GRBs at high redshift to a maximum of $14 \%(5 \%)$ for $z>6(z>7)$. The mean redshift of the host sample is assessed to be $\langle z\rangle \gtrsim 2.2$. Using this more complete sample, we confirm previous findings that the GRB rate at high redshift $(z \gtrsim 3)$ appears to be in excess of predictions based on assumptions that it should follow conventional determinations of the star formation history of the universe, combined with an estimate of its likely metallicity dependence. This suggests that either star formation at high redshifts has been significantly underestimated, for example due to a dominant contribution from faint, undetected galaxies, or that GRB production is enhanced in the conditions of early star formation, beyond that usually ascribed to lower metallicity.
\end{abstract}

Gamma-Ray Bursts 2012 Conference-GRB2012,

May 07-11, 2012

Munich, Germany 


\section{Introduction}

We have secured GRB host galaxy information for a homogeneous sample of 69 Swift GRBs with a large program at the VLT [1]. The sample has been carefully selected and obeys strict and well-defined criteria. To optimize the survey, we focused on systems with the best observability, which also have the best available information. The main results of The Optically Unbiased GRB Host (TOUGH) survey is presented in [2-5], including fundamental properties of the hosts, Ly $\alpha$ emission and new redshifts.

Here we present the TOUGH campaign for missing redshifts via VLT/FORS [3] and VLT/Xshooter [5]. We attempted spectroscopic observations of most TOUGH host candidates with $R \lesssim$ 25.5 mag that did not have a reported reliable redshift.

\section{Redshift Measurements and Constraints}

We have obtained 15 new host redshifts; Fig.1 shows the cumulative redshift distribution of the 53 TOUGH bursts with a measured redshift. Also plotted is a conservative systematic error band (hatched region) containing information for all the 69 TOUGH bursts. The shaded region represents the likely statistical ( $1 \sigma$ standard error of the sample) uncertainty of the measured redshift distribution under the assumption that it is a true random sample of the overall population. The sampling error and the conservative systematic error region are shown separately to clearly illustrate that incompleteness dominates the sample, and more is gained by reducing the systematics rather than increasing the sample size. Using both error regions we can set a conservative limit on the maximum number of Swift bursts at $z>6(z>7): 14 \%$ (5\%).

The average (median) redshift of the 53 TOUGH bursts is $\langle z\rangle=2.23(\tilde{z}=2.14)$, significantly lower than the early Swift results indicated [6]. This difference may simply reflect the comparatively small samples analyzed in that paper, but could also be due to an increased success in measuring redshifts $z<2$ using weaker absorption lines in afterglow spectra, and via host galaxies. The mean redshift could be as low as $\langle z\rangle \sim 1.7$ (upper boundary of the hatched region) although it is unlikely that the majority of bursts with unknown redshifts would be located at very small distances. In fact, it is more probable that $\langle z\rangle \gtrsim 2.2$ since we have only targeted the brightest galaxies in the sample $(R \lesssim 25.5 \mathrm{mag})$ for spectroscopic follow-up.

\section{Modelling}

Illustrative model fits are presented in [3] and described in detail there. We assume that the GRB rate follows the star-formation rate (SFR) history, and consider two different SFR history parameterizations which we label as follows. SFR1 is an update [7] of the SFR history models of [8] to include recent data from [9] and [10], combined with a low-metallicity modification following the prescription of [11]. SFR2 is model A from [12] which represents a SFR history which remains constant beyond $z \sim 3$. It may, for example, be considered a more extreme low-metallicity correction to the cosmic SFR. Or it may represent a correction $[13,14]$ to the high-redshift SFR as estimated from flux-limited surveys by the integration of galaxy luminosity functions (LFs) thus obtained. This would be due to a large amount of hidden star formation in faint, low-mass, and high specific SFR galaxies of the type that GRBs tend to be associated with at lower redshift. 


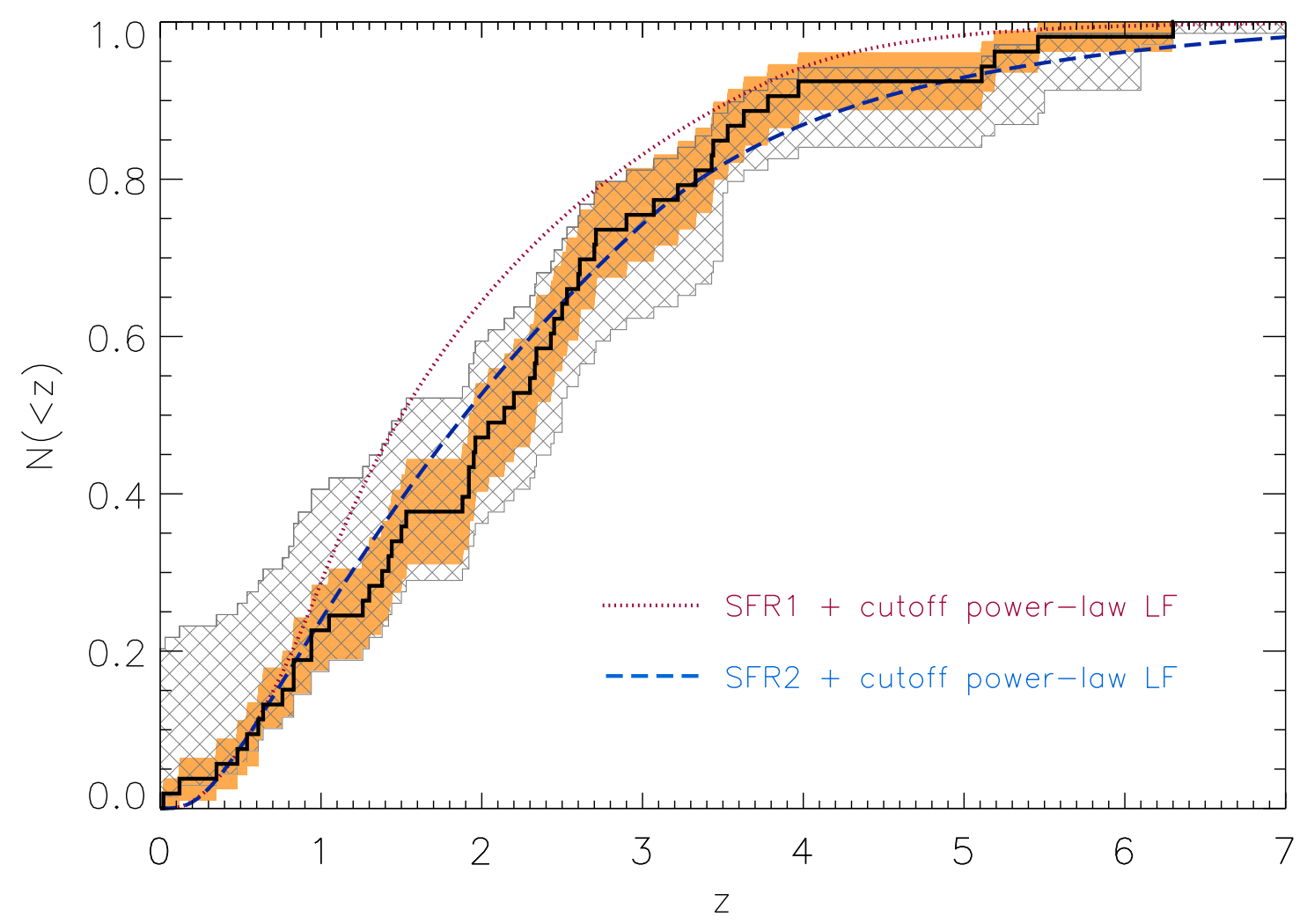

Figure 1: Thick solid curve: the cumulative fraction of GRBs as a function of redshift for the 53 Swift bursts in the TOUGH sample with a measured redshift $(\langle z\rangle=2.23)$. Hatched region: this is a conservative error region showing the systematic error on the thick solid curve. Shaded region: statistical region showing the $1 \sigma$ sampling error band around the thick solid curve. Dotted curve: the expected redshift distribution for Swift observable long GRBs using the SFRl history parameterization, i.e. the canonical SFR history discussed in [8] (see the main text). Dashed curve: the same redshift distribution for the SFR2 history parameterization, i.e. a model where the SFR history remains constant beyond $z \sim 3$ [12] (see the main text). From [3].

Modeling is performed in the standard manner [15] to produce $\log N-\log L$ number count distributions for various parameters of the LF, which are then fit by $\chi^{2}$ minimization to the observed $\log N-\log L$ distribution of all Swift bursts with peak photon flux $>1 \mathrm{~cm}^{-2} \mathrm{~s}^{-1}$. We emphasize that the redshift distribution is not part of this fitting procedure, but is always purely a result. In Fig. 1, we plot the redshift distributions from our best fitting models in comparison to the TOUGH redshift data.

At face value, these results seem to imply that GRBs follow a cosmic SFR history that is significantly enhanced at high redshift compared to estimates from flux-limited surveys. Given what is known about GRB hosts, it is entirely feasible that GRBs trace star formation at high redshift that would be undetectable by other means. It is of course also possible that the simple low-metallicity enhanced SFR parameterization used in the SFRI model is inadequate, or that the LF could have a more complex form and/or evolve with redshift. It should also be noted that [17] find that there is no strong preference for a metallicity cut. 


\section{Discussion}

It is possible that star formation at high redshifts has been significantly underestimated. Even at $z \sim 2$ it appears that the galaxy LF has a substantially steeper faint-end slope than locally [16], while recent LF studies in the Hubble Ultra-Deep Field have concluded that at $z \gtrsim 7$ so-far undetected galaxies are likely to completely dominate the total star formation activity [18, 19]. Alternatively, it could be that GRB production is substantially enhanced in the conditions of early star formation, beyond the metallicity-dependent rate correction already applied. In the long run, large complete samples of GRB redshifts should shed light on whether the GRB rate is proportional to SFR or whether other effects play an important role.

We have now reached a point in GRB research where a single burst rarely elucidates and illuminates our general understanding of the field. It is important to focus on well-defined samples and population studies, where systematics and biases can be minimized. Swift has made it possible to build such a sample and thanks to new available instrumentation, such as the VLT/X-shooter [20], we can continue to follow this track into the future.

\section{References}

[1] J. Hjorth, et al., The Optically Unbiased GRB Host (TOUGH) Survey. I. Survey Design and Catalogs, ApJ, submitted (2012) [arXiv: 1205.3162].

[2] D. Malesani, et al., The Optically Unbiased GRB Host (TOUGH) Survey. II. Fundamental Properties, ApJ, in prep.

[3] P. Jakobsson, et al., The Optically Unbiased GRB Host (TOUGH) Survey. III. Redshift Distribution, ApJ 752 (2012) 62 [arXiv: 1205.3490].

[4] B. Milvang-Jensen, et al., The Optically Unbiased GRB Host (TOUGH) Survey. IV. Ly $\alpha$ Emitters, ApJ, in press (2012) [arXiv: 1205.3779].

[5] T. Krühler, et al., The Optically Unbiased GRB Host (TOUGH) Survey. V. VLT/X-shooter Emission-Line Redshifts for Swift GRBs at $z \sim 2$, ApJ, submitted (2012) [arXiv: 1205.4036].

[6] P. Jakobsson, et al., A Mean Redshift of 2.8 for Swift Gamma-Ray Bursts, A\&A 447 (2006) 897 [arXiv: 0509888].

[7] L.-X. Li, Star formation history up to $z=7.4$ : implications for gamma-ray bursts and cosmic metallicity evolution, MNRAS 388 (2008) 1487 [arXiv: 0710 . 3587].

[8] A. M. Hopkins \& J. F. Beacom, On the Normalization of the Cosmic Star Formation History, ApJ 651 (2006) 142 [arXiv: 0601463 ].

[9] R. J. Bouwens, et al., $z \sim 7-10$ Galaxies in the HUDF and GOODS Fields: UV Luminosity Functions, ApJ 686 (2008) 230 [arXiv: 0803.0548$].$

[10] N. A. Reddy, et al., Multiwavelength Constraints on the Cosmic Star Formation History from Spectroscopy: the Rest-Frame Ultraviolet, $H \alpha$, and Infrared Luminosity Functions at Redshifts $1.9<z<3.4$, ApJS 175 (2008) 48 [arXiv : 0706.4091 ]

[11] N. Langer \& C. A. Norman, On the Collapsar Model of Long Gamma-Ray Bursts:Constraints from Cosmic Metallicity Evolution, ApJ 638 (2006) L63 [arXiv : 0512271]. 
[12] M. Schmidt, Gamma-Ray Burst Luminosity Functions Based on a Newly Discovered Correlation Between Peak Spectral Energy and V/V $V_{\max }$, ApJ 700 (2009) 641 [arXiv: 0905.2968 ].

[13] M. D. Kistler, et al., The Star Formation Rate in the Reionization Era as Indicated by Gamma-Ray Bursts, ApJ 705 (2009) L104 [arXiv: 0906 . 0590].

[14] F. J. Virgili, et al., Gamma-ray burst rate: high-redshift excess and its possible origins, MNRAS 417 (2011) 3025 [arXiv: 1105.4650$]$.

[15] D. Guetta \& T. Piran, Do long duration gamma ray bursts follow star formation?, JCAP 7 (2007) 3 [arXiv:0701194].

[16] N. A. Reddy \& C. C. Steidel, A Steep Faint-End Slope of the UV Luminosity Function at z $\sim 2-3$ : Implications for the Global Stellar Mass Density and Star Formation in Low-Mass Halos, ApJ 692 (2009) 778 [arXiv:0810.2788].

[17] J. Elliott, et al., The long $\gamma$-ray burst rate and the correlation with host galaxy properties, A\&A 539 (2012) 113 [arXiv:1202.1225].

[18] R. J. Bouwens, et al., Lower-luminosity Galaxies Could Reionize the Universe: Very Steep Faint-end Slopes to the UV Luminosity Functions at z>5-8 from the HUDF09 WFC3/IR Observations, ApJ 539 (2012) L5 [arXiv: 1105.2038$].$

[19] N. R. Tanvir, et al., Star formation in the early universe: beyond the tip of the iceberg, ApJ, in press (2012) [arXiv:1201.6074].

[20] J. Vernet, et al., X-shooter, the new wide band intermediate resolution spectrograph at the ESO Very Large Telescope, A\&A 536 (2011) 105 [arXiv: 1110 . 1944]. 